\title{
Anti-PSMA/CD28 Bispecific Antibody REGN5678
}

National Cancer Institute

\section{Source}

National Cancer Institute. Anti-PSMA/CD28 Bispecific Antibody REGN5678. NCI

Thesaurus. Code C162936.

\begin{abstract}
A bispecific antibody directed ag ainst both the tumor-associated antigen (TAA) prostatespecific membrane antigen (PSMA) and the co-stimulatory T-cell-specific surface glycoprotein CD28, with potential immunostimulating and antineoplastic activities. Upon administration of anti-PSMA/CD28 bispecific antibody REGN5678, this bispecific antibody binds to both CD28 on cytotoxic T-lymphocytes (CT LS) and PSMA found on PSMAexpressing tumor cells. This activates and redirects CT Ls to PSMA-expressing tumor cells, which may result in the CT L-mediated cell death of PSMA-expressing tumor cells. PSMA is overexpressed on the surface of metastatic and hormone-refractory prostate cancer cells.
\end{abstract}

